\title{
Aplicación de una técnica cualitativa para la elaboración de un plan de atención al paciente pluripatológico en hemodiálisis
}

\author{
$M^{a}$ Dolores Abril Sabater \\ Ruben Iglesias Sanjuón \\ Almudena Jerez Garcia \\ María J. López Parra \\ Núria Mañé Buixó \\ Elios Yuste Jiménez
}

Servicio de Nefrología
Corporació Parc Taulí
Sabadell (Barcelona)

\section{RESUMEN}

En nuestra unidad de nefrología han aumentado los pacientes en programa de hemodiálisis crónica, muchos de ellos son de edad avanzada, y esto ha implicado un aumento de trabajo organizativo y asistencial. Se ha detectado la necesidad de una organización y planificación más detallada para atender al paciente pluripatológico de forma integral, dado que actualmente no existe una línea de trabajo bien definida y esto provoca algunas deficiencias, tensión en el equipo, burocracia excesiva y/o repetida, y genera malestar en el paciente y su entorno.

Con el objetivo de dar una atención integral con criterios de efectividad y eficiencia a los pacientes pluripatológicos en hemodiálisis, se ha analizado el método de trabajo actual para diseñar un plan de atención integral al paciente, utilizando la técnica Delphi.

Los pacientes tratados en diálisis crónica durante el 2002 fueron 184, predominando las siguientes características: edad avanzada (69 \pm 13 años), con otras patologías asociadas como hipertensión, cardiopatías, diabetes o vasculopatías. Durante este año han fallecido 19 pacientes (14,28\%).

\section{Correspondencia:}

$M^{\underline{a}}$ Dolores Abril Sabater

C/ Parc Taulí s/n

08208 Sabadell (Barcelona)

Teléfono: 937458423

E-mail:eyuste@cspt.es
El uso de la técnica Delphi ha sido útil para identificar las necesidades de los pacientes pluripatológicos y diseñar una buena planificación y organización para su atención, aportando una mejora de la calidad asistencial y consiguiendo una mayor satisfacción de los pacientes y de los profesionales.

PALABRAS CLAVE: HEMODIÁLISIS

TÉCNICA DELPHI

PATOLOGÍAS ASOCIADAS

ATENCIÓN INTEGRAL

\section{APPLICATION OF A QUALITATIVE TECHNIQUE FOR PREPARING AN ATTENTION PLAN FOR PA- TIENTS WITH MULTIPATHOLOGICAL PROBLEMS UNDER HAEMODIALYSIS}

\begin{abstract}
In our nephrology unit there has been an increase in the number of patients on chronic haemodialysis programmes; many are elderly and this has supposed an increase in the organisational and assistential work. The need has been detected for more detailed organisation and planning to comprehensively attend patients with multipathological problems, as there is currently no well-defined line of work and this causes some shortfalls, tension among the team, excessive and/or repeated bureaucracy and generates unrest in the patients and their surroundings.
\end{abstract}


In order to give integral attention with criteria of effectiveness and efficiency to patients with multipathological problems under haemodialysis, the current working method has been analysed to draw up a plan of integral attention for patients, using the Delphi technique.

184 patients were treated in chronic dialysis in 2002 , with a predominance of the following characteristics: elderly $(69 \pm 13$ years of age), with other associated pathologies such as hypertension, caryopathy, diabetes or vasculopathy. This year 19 patients died (14.28\%).

The Delphi was useful for identifying the needs of patients with multipathological problems and for drawing up a good plan and organisation for their care, giving an improvement in the quality of the assistan$\mathrm{ce}$ and achieving greater satisfaction from patients and professionals.

\section{KEYWORDS: HAEMODIALYSIS}

DELPHI TECHNIQUE

ASSOCIATED PATHOLOGIES

INTEGRAL ATTENTION

\section{INTRODUCCIÓN}

Una gran parte de los enfermos que tratamos en las unidades de HD sufren más de una enfermedad y, a su vez, cada una le afecta a más de un órgano. Se estima en cinco la media de problemas médicos de un paciente y sólo el 15\% tienen un solo problema médico definido. En estudios de comorbilidad en ingresos hospitalarios de mayores de 65 años se ha comprobado que tienen un promedio de 6,4 enfermedades crónicas. Por otra parte, los estudios necrópsicos han confirmado que la presencia de pluripatología objetiva es incluso superior a lo que indican los datos obtenidos en las encuestas de salud.

El término "enfermo pluripatológico" se utiliza asiduamente al referirnos a ancianos frágiles, a enfermos polimedicados, a pacientes hiperfrecuentadores, o a reingresadores en los hospitales y, aunque no es sinónimo de ninguno de estos conceptos, constituye el núcleo esencial de cada una de estas realidades.

La sala de hemodiálisis donde se desarrolla nuestro estudio pertenece al Servicio de Nefrología de un hospital comunitario, con una población de referencia de 387.000 habitantes, y son muchos los pacientes que cumplen las características mencionadas.

Hemos detectado la necesidad de una organización y planificación más detallada para atender al paciente pluripatológico de forma integral, dado que actualmente no existe una línea de trabajo bien definida y esto provoca algunas deficiencias: tensión en el equipo, burocracia excesiva y/o repetida, complicaciones en el estado general del paciente, y malestar en el paciente y su entorno.

Ya que los estudios realizados sobre este tema no son concluyentes, la investigación cualitativa, y, dentro de ella, la técnica Delphi, pueden ser útiles para profundizar en el estudio de las necesidades del paciente pluripatológico.

\section{OBJETIVOS}

1- Analizar el método de trabajo actual.

2- Diseñar un plan de atención al paciente pluripatológico, evaluando la utilidad de la técnica de Delphi.

\section{PACIENTES Y MÉTODO}

\section{Pacientes}

Los pacientes tratados en diálisis crónica durante el año 2002 fueron 184, que presentaban las siguientes características:

- Edad avanzada (con una media 69+ 13 años).

- Predominio de hombres 98 (78\%), frente a las mujeres 28 (22\%).

- Frecuente existencia de un factor de riesgo asociado o de otras enfermedades, destacando: hipertensión, cardiopatías, diabetes y vasculopatías. Nuestros pacientes tienen una media de 4,6 enfermedades asociadas, 110 de ellos presentan más de 3 patologías asociadas, por tanto, tenemos un $87,3 \%$ de enfermos pluripatológicos.

- 29 de ellos están en tratamiento psiquiátrico.

- 15 están siendo asistidos por la Trabajadora Social.

- Situación respecto al trasplante renal: 26 (20,5\%) de los pacientes de hemodiálisis están en lista de espera para trasplante renal; 13 (11,48\%) pendientes de completar estudios; excluidos por edad, 40 (31,97\%) excluidos por patología asociada 42 (32,79\%), excluidos por decisión propia 5 (4,10\%).

- Durante este año han fallecido 19 pacientes que estaban en programa de hemodiálisis, lo cual supone un $14,28 \%$ del total (tasa dentro de los límites normales en nefrología). Las causas de muerte están asociadas principalmente a: cardiopatías $36,8 \%$, problemas vasculares $21,05 \%$, infecciones $15,8 \%$, hepatopatologías $10,52 \%$ y neoplasias $10,52 \%$. 


\section{Método}

La metodología utilizada ha sido:

- Descriptiva, para describir el método actual de trabajo, además se ha tratado de una observación participante.

- Para desarrollar la propuesta de atención al paciente pluripatológico en hemodiálisis, se ha utilizado la Técnica de Delphi entre los profesionales de la Unidad. Ésta es una técnica de investigación cualitativa, que consiste en tres cuestionarios por escrito, espaciados en el tiempo, por al menos una semana, a un grupo de individuos implicados en la situación, que tengan interés por el tema y tengan capacidad para expresar por escrito sus opiniones.

La técnica Delphi se ha desarrollado de la siguiente forma: se hizo la descripción del problema y se redactó el primer cuestionario junto con una carta de presentación. En este primer cuestionario, se pedía una lista de soluciones para afrontar el problema y que se aportaran los comentarios oportunos. Este primer cuestionario se entregó a la totalidad del personal asistencial de Nefrología. Se realizó un análisis de resultados (agrupación de ideas y comentarios).

Con el segundo cuestionario, se envió un resumen de los resultados obtenidos en el primero, con todos los temas que se habían propuesto, así como los comentarios. De éstos, debían escoger 10 y ordenarlos por orden de importancia decreciente. Este segundo cuestionario se entregó junto con una carta de agradecimiento. Se creó una base de datos para el análisis del segundo cuestionario.

En el tercer cuestionario se entregaron los 10 temas más puntuados, ordenados según importancia, así como los comentarios recogidos. Se entregó para que, nuevamente, colocaran estos diez temas por orden de importancia decreciente. Se analizaron los resultados en una base de datos y, a partir de éstos, se elaboró un informe final y el diseñó del plan de atención al paciente pluripatológico.

\section{RESULTADOS}

\section{Análisis de la organización actual del trabajo}

Actualmente, a cada paciente se le cita para una Consulta de Enfermería con el fin de realizar un programa educativo dirigido a los siguientes temas: ubicación general y funcionamiento del servicio, dieta para hemodiálisis, cuidado del acceso vascular, y se intenta resolver todas las dudas que plantee el paciente.
Los 184 pacientes acuden para recibir tratamiento dialítico tres veces por semana, repartidos en tres turnos. El personal de enfermería trabaja en dos turnos, mañana y tarde; por tanto atiende a un turno y medio de pacientes.

Para atenderlos contamos con:

- Nefrólogos: 1 Responsable médico, 8 adjuntos de nefrología.

- Profesionales de enfermería: 1 Responsable de enfermería, 1 coordinadora de enfermería, 30 diplomados en enfermería (a tiempo completo y parcial), 13 auxiliares de enfermería.

- Personal administrativo: 1

- Técnicos de mantenimiento: 3

- Personal de limpieza: 6

Respecto a enfermería, actualmente el ratio de pacientes por enfermera es de 3'1 pacientes por turno de diálisis. Desde hace un año, se trabaja por sistema de enfermera de referencia. Cada enfermera es responsable del seguimiento y plan de cuidados de 3 pacientes de cada turno y de conectar y desconectar los pacientes que tiene asignados, controlar la sesión de hemodiálisis (prevenir complicaciones), realizar las curas de enfermería que requiera y administrar tratamientos farmacológicos.

El ciclo de seguimiento de cada grupo de pacientes es rotativo y dura 12 semanas. Al tratarse de pacientes crónicos que acuden tres veces por semana a recibir tratamiento, se pensó que podía ser positivo crear un sistema rotativo para evitar dependencia del profesional y evitar sobrecarga psicológica en los profesionales. El resto de tareas de la sala se realizan en equipo junto con la auxiliar de enfermería. Existe un responsable de enfermería que con la ayuda de un coordinador se encargan de organizar y planificar el funcionamiento del servicio.

\section{Resultado de la aplicación de la Técnica de Delphi} Los resultados del primer cuestionario fueron agrupados en un total de 17 temas, cada uno de los cuales contenía varios ítems. Los temas eran los siguientes: derivar a consultas externas, derivar a atención primaria, comunicación externa, comunicación interna, recursos materiales en hemodiálisis, recursos materiales en hospital de agudos, recursos humanos, seguimiento del paciente, trabajo en equipo multidisciplinar, formación del personal, educación del paciente, objetivos realistas, sesiones con otras especialidades, organización externa, organización interna, responsabilidad, grupos de trabajo. 
Estos resultados fueron los utilizados para la elaboración del segundo cuestionario, de los cuáles se debían elegir diez temas por orden de importancia. Los seleccionados fueron: comunicación externa, seguimiento del paciente, trabajo en equipo multidisciplinar, recursos humanos, responsabilidad, objetivos, comunicación interna, educación del paciente, recursos materiales en hemodiálisis, recursos materiales en hospital de agudos.

Y finalmente en el tercer cuestionario se propuso puntuar los diez temas que obtuvimos con el segundo cuestionario por orden de importancia. A partir de estos resultados, se propuso el siguiente plan por orden de prioridad:

\section{1) Seguimiento de paciente:}

Realizar una valoración exhaustiva del paciente cuando inicia hemodiálisis mediante la utilización de un test a elegir entre: Barthel, Lawton y Brody, escala de valoración social de Gijón. Valorar también la posibilidad de utilizar las trayectorias clínicas que se están elaborando en nuestro hospital.

\section{2) Comunicación externa:}

En cuanto a la mejora de la comunicación externa, se considera importante disponer de información informatizada (por ejemplo vía e-mail) para poder acceder a toda la información que permita dar al paciente una correcta atención. También se considera importante para esta mejora establecer una comunicación fluida con el cuidador principal del paciente pluripatológico una vez cada doce semanas.

\section{3) Trabajo en equipo multidisciplinar}

Crear un plan de cuidados de atención individualizada en coordinación con los facultativos. Realizar sesiones una vez al mes, donde se expondrían casos clínicos de los pacientes con varias patologías, evaluando su estado. Pasar visita conjuntamente, una vez por semana, el médico y la enfermera de referencia.

\section{4) Responsabilidad}

Buscar mecanismos para motivar la implicación y responsabilidad individual de los profesionales respecto a los pacientes asignados.

\section{5) Comunicación interna}

Centralizar toda la información clínica en un solo documento informatizado para el seguimiento del paciente y mejorar la comunicación entre los profesionales (que haya más retroalimentación entre éstos).

\section{6) Recursos humanos}

Identificar las necesidades en lo que se refiere a recursos humanos (camillero propio, refuerzo de secretaria, tra- bajadoras sociales, ampliar personal de enfermería, psicólogo que de soporte tanto a los pacientes como al personal sanitario).

\section{7) Educación al paciente}

Dentro de la consulta de enfermería hacer mayor hincapié para que los pacientes sepan a quién se ha de dirigir dependiendo del problema que tenga (médico de familia, nefrólogo, trabajadora social, personal de enfermería, personal administrativo).

\section{8) Recursos materiales en hemodiálisis}

Identificar necesidades en cuanto a material fungible y bienes materiales (carro de curas, camas automáticas, tubo neumático).

\section{9) Objetivos}

Que los profesionales, en la medida de lo posible, participen en la elaboración de los objetivos de la unidad, siempre buscando que éstos sean alcanzables.

\section{0) Recursos materiales en hospital de agudos} Identificar las posibles mejoras del hospital de día y ampliación de la unidad de hospitalización.

\section{DISCUSIÓN}

Con este trabajo hemos conseguido hacer una reflexión de la forma en que son atendidos actualmente los pacientes pluripatologicos en HD. Esto nos ha ayudado a conseguir establecer una metodología de trabajo que unifique y sistematice la atención a este tipo de pacientes. También se favorece el hecho de trabajar mediante el sistema de equipo multidisciplinar, lo que, a su vez, permite la comunicación entre los distintos servicios que se encarguen del seguimiento y tratamiento de un mismo paciente.

Hemos comprobado que la técnica de Delphi puede ser una buena herramienta para la solución de conflictos, especialmente en equipos de trabajo muy numerosos, en los que llegar al consenso por otras vías puede ser complejo. Enfermería tiene un papel muy relevante para identificar las necesidades del paciente pluripatológico y para integrar y coordinar su atención. Por todo ello, técnicas como la expuesta puede ser de gran utilidad para ofrecer una atención de calidad.

En ocasiones, nuestro trabajo se vuelve rutinario y se nos pueden escapar los detalles. Es importante, pues, analizar nuestros métodos de trabajo periódicamente y realizar los cambios que sean necesarios. 
Desde hace ya varios años, las características de la población en hemodiálisis crónica están variando. Debemos, por lo tanto, adaptar nuestra organización y gestión a sus necesidades. El uso de la técnica de Delphi nos ha aportado ventajas de libertad de expresión, reflexión individual, posibilidad de contrastar entre los diferentes cuestionarios, evitar desplazamientos, reuniones de todo el equipo y pérdida de tiempo en discusiones. Aunque presenta algunos inconvenientes: requiere capacidad de expresión escrita, el consenso puede hacer que se rechacen buenas nuevas ideas, importante labor de análisis y síntesis.

\section{CONCLUSIÓN}

El analizar el método de trabajo actual y utilizar la Técnica de Delphi, como paso previo para diseñar un plan de atención al paciente pluripatólogico ha sido útil para poder identificar las necesidades de estos pacientes y a su vez con los resultados obtenidos, pensamos que se puede facilitar la gestión y organización de la unidad para conseguir así un funcionamiento óptimo de la misma.

\section{BIBLIOGRAFÍA}

1. Consejería de Salud de la Junta de Andalucía. Atención al paciente pluripatológico, proceso asistencial integrado. Sevilla 2002

2. Dee Ann G. Asignación de enfermería por grupos de pacientes. Gestión de Enfermería. Barcelona: Masson-Salvat, Enfermería; 1994.

3. García R, Amezcua C. Técnicas cualitativas de la investigación. Documentación social. UNED.

4. Klahr S. Anemia, diálisis and dollars. N Engl Med 1996; 334:461-463.

5. Memoria del Servicio de Nefrología del año 2002. Hospital Parc Taulí. Sabadell. Barcelona.

6. Rota L et al. Diseño de una consulta de enfermería. Educación a los pacientes en hemodiálisis. Libro de comunicaciones XXVII Congreso Nacional de la Sociedad Española de Enfermería Nefrológica, Bilbao 2002; 91-96.

Enlaces de interés:

http://www.jomozabal.com/pluripat.htm 